\title{
Platelet to lymphocyte and neutrophil to lymphocyte ratios in endometrial pathologies
}

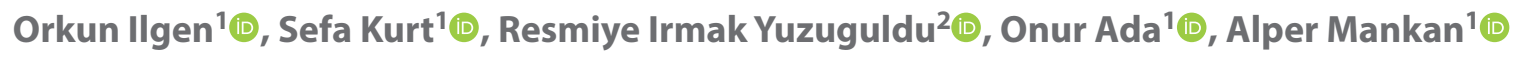 \\ ${ }^{1}$ Department of Obstetrics and Gynecology, 9 Eylul University School of Medicine, Izmir, Turkey \\ ${ }^{2}$ Department of Obstetrics and Gynecology, Dokuz Eylul University, Medical Faculty, Turkey
}

\begin{abstract}
Objectives: Aim of this study was to evaluate the relationship between platelet to lymphocyte ratio (PLR), neutrophil to lymphocyte ratio (NLR), and endometrial pathologies.

Material and methods: The database of our institution was reviewed. Cases with endometrial pathology including endometrial cancer $(E C)$, endometrial hyperplasia with atypia and without atypia, normal endometrial findings, between January 2015 to January 2020, were collected. Their CBC results and clinicopathologic data were determined. The relation between the platelet-lymphocyte ratio (PLR), neutrophil-lymphocyte ratio (NLR), and endometrial pathologies was evaluated.

Results: NLR was significantly higher in patients with endometrial cancer compared to other endometrial pathologies including endometrial hyperplasia with and without atypia and patients with normal endometrial findings. NLR cut-off value was determined 3.55 to discriminate cancer among other endometrial pathologies. PLR had not a significant difference between the endometrial pathologies.
\end{abstract}

Conclusion: NLR seems to be an effective and simple marker to discriminate endometrial cancer among endometrial pathologies by contrast with PLR.

Key words: neutrophil to lymphocyte ratio; platelet to lymphocyte ratio; endometrial cancer; endometrial pathologies

Ginekologia Polska

\section{INTRODUCTION}

Inflammation and related parameters have a crucial role in cancer development and spread [1]. Relation between inflammation and cancer is a focused issue for investigators to explain the interaction between them. However, precise pathophysiologic mechanisms have not been definitely determined yet. It is a well-known fact that there is mutual induction between cancer and inflammation [2]. Endometrial hyperplasia $(\mathrm{EH})$ which is usually a result of continuous unopposed estrogen exposure is a predisposing factor for the development of endometrial carcinoma (EC) with rates of up to $30 \%$ [3]. Relation between inflammation markers and cancer progression was investigated previously in different studies. Neutrophil-lymphocyte ratio (NLR) was determined as an inflammation marker. Increased neutrophil to lymphocyte ratio was found to be related to worse overall-survival in EC and malign mesothelioma patients [4,5]. Platelet-lymphocyte ratio $(P L R)$ is another parameter that was used to evaluate the prognosis of certain cancer types including colorectal and EC
$[4,6]$. Hereby, a cheap, easy, simple, and reproducible marker is a need to distinguish the endometrial pathologies including normal, hyperplasia without atypia, hyperplasia with atypia, and endometrial cancer. To our knowledge, there has not been any study that aims to distinguish all four endometrial pathologies by using all three parameters including NLR and $P L R$. The present study aims to investigate the association between NLR, PLR, and endometrial pathologies.

\section{MATERIAL AND METHODS Study population}

Clinicopathological data of the patients with the diagnosis of EC, EH (with and without atypia), normal endometrial results (proliferative, endometrium, secretuar endometrium, atrophic endometrium, endometrial cells) as a result of endometrial biopsy who applied to the Dokuz Eylul University Hospital from January 2015 to January 2020 were collected from the hospital database retrospectively. The ethics committee approval was obtained for this study from the local ethics committee of Do- 
Table 1. Clinicopathologic data of the patients among groups

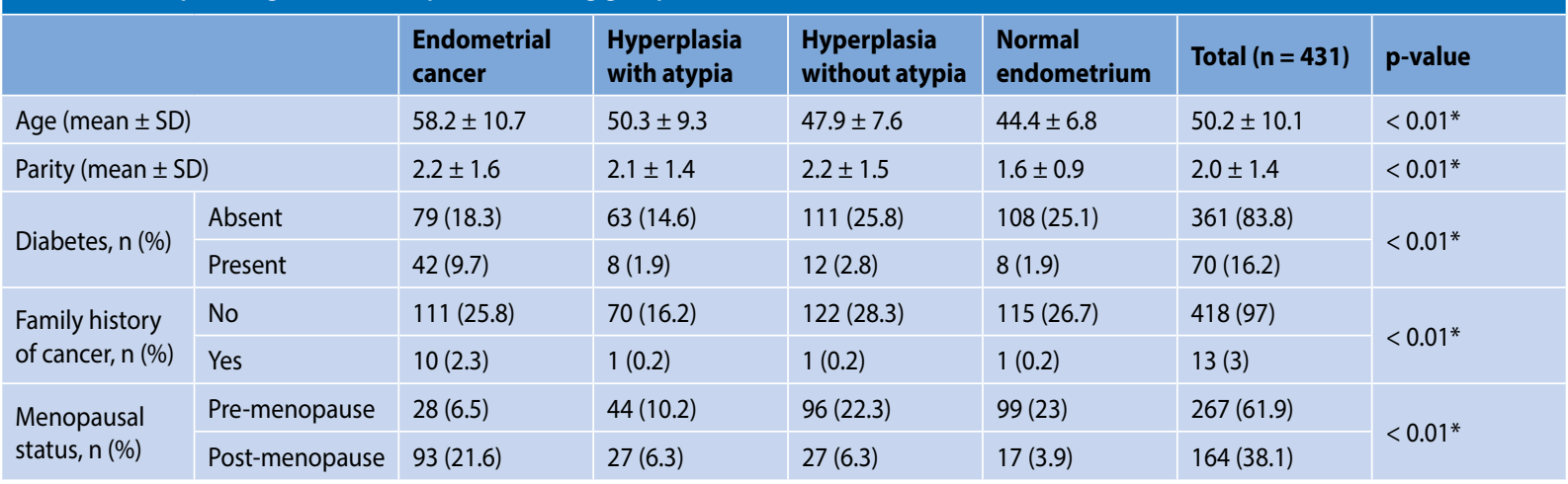

SD — standard deviation

kuz Eylul University. Eligible patients had undergone detailed gynecological examination and preoperative assessment including radiological imaging, histopathological examination of endometrial biopsy, and full blood count (FBC). Patients whose FBC assessment two weeks before surgery were excluded from the study. Besides, patients with insufficient clinicopathological data, irregular follow-up information, with granulocyte colony-stimulating factor use, inflammatory disease, carcinoid tumor, other malignancies or disorders that affect hematologic parameters, or those that were given a blood transfusion during the last two weeks before the blood sampling or treated with radiotherapy and chemotherapy were excluded.

\section{Data collection}

The hospital's electronic medical record database was used to obtain patients' clinicopathological data: (i) Basic information including age, menopause age, reproductive history, and comorbidities; (ii) FBCs including neutrophil, lymphocyte, and platelet counts were expressed in $\times 10^{9} / \mathrm{L}$. NLR and PLR were calculated as the absolute neutrophil, and platelet count divided by the absolute lymphocyte count, respectively. Pathologic results of the endometrial biopsies were classified by the World Health Organization 2014 classification system which divides hyperplasia into two categories defined as hyperplasia with atypia and hyperplasia without atypia.

\section{Statistical analysis}

The normality of distributions of the variables was determined by the Kolmogorov-Smirnov test. Continuous variables were presented as mean \pm standard deviation whether it had a normal distribution. However, variables without normal distribution were presented as median values (minimum-maximum). Association between continuous and categorical variables was evaluated by one-way ANOVA or Kruskal-Wallis test and additionally, Mann-Whitney $U$ test was used for further evaluation of the preliminary results. Post-hoc tests were performed to determine the group which led to statistically significant differentiation among the four groups. Univariable and multivariable analyses were used to estimate the odd's ratio (OR) and 95\% confidence intervals ( $\mathrm{Cls}$ ) between variables. Receiver operating characteristics (ROC) curve analysis was used to calculate a cut-off value to distinguish groups from each other. The comparison of proportions was calculated by the Chi-square test. P-value $<0.05$ was considered statistically significant. All analyses were performed by using IBM SPSS Statistics Version 25.

\section{RESULTS}

The mean age of the patients was $50.2 \pm 10.1$ years. The mean age of the EC patients was found $58.2 \pm 10.7$ years. EC patients were older than the other groups of the patients ( $p<0.01$ ), however, no significant difference was found between hyperplasia with atypia and without atypia groups. Postmenopausal patients, family history of cancer, and diabetes were determined higher in the EC patients as expected ( $p<0.01 ; p<0.01 ; p<0.01$, respectively). Clinicopathologic data of the patients were summarized in Table 1.

Median neutrophil to lymphocyte ratio of endometrial cancer, hyperplasia with atypia, hyperplasia without atypia, and patients with normal endometrial findings groups were 2.4 (0.7-12.2), 2.1 (0.01-18), 2.1 (0.1-148), and 2 (0.4-6); respectively. The neutrophil to lymphocyte ratio was found significantly higher in the endometrial cancer patients rather than other groups of the patients $(p=0.04)$. ROC curve analysis was performed to determine the cut-off value for the NLR. NLR cut-off value to distinguish endometrial carcinoma among endometrial pathologies was determined 3.55 with the highest Youden index [likelihood ratio $(\mathrm{LR})=2.65$ ] (area under the curve, $A U C=0.587$ ) (Fig. 1). Median platelet to lymphocyte ratio of endometrial cancer, hyperplasia with atypia, hyperplasia without atypia, and patients with normal endometrial findings groups were 137.6 (54.2-476.6), 126.8 (37.5-391.8), 125.2 (55.1-626), and 133.8 (15.5-363.9), respectively. There was no significant difference between groups in terms of PLR $(p=0.84)$. Associa- 


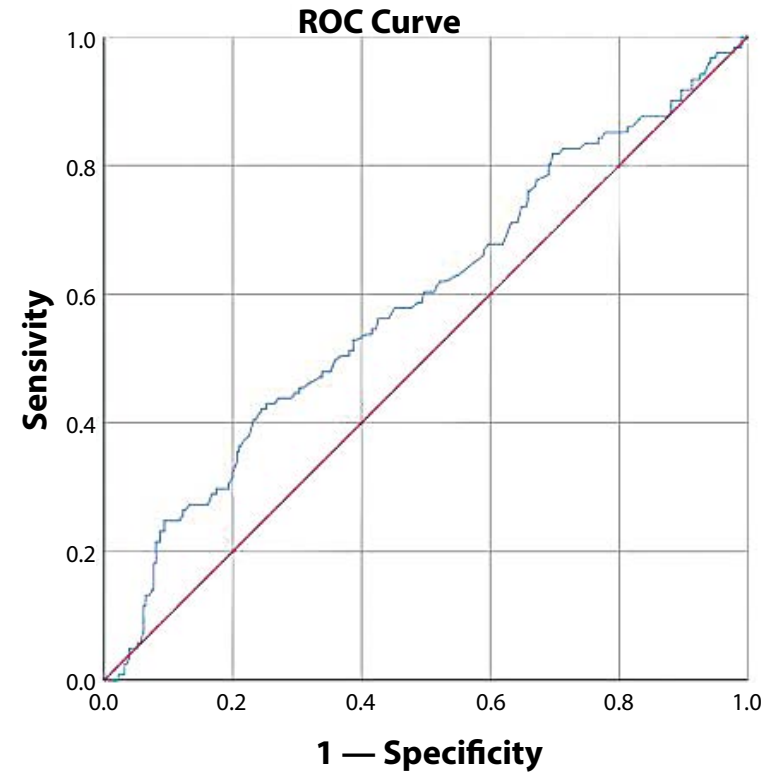

Diagonal segments are produced by ties

Figure 1. ROC curve analysis of the NLR for endometrial cancer in endometrial pathologies; NLR - neutrophil to lymphocyte ratio; ROC - receiver operating characteristics tions between NLR, PLR, and endometrial pathologies were summarized in Table 2. Besides, endometrial pathologies were divided into subgroups by histopathological tumor grade and The International Federation of Gynecology and Obstetrics (FIGO) staging in itself, then compared with non-cancerous pathologies including $\mathrm{EH}$ (with or without atypia) and normal endometrial results in terms of NLR and PLR. It was found that grade 1 and grade 3 EC patients had significantly higher NLR compared to patients with non-cancerous endometrial pathologies including $\mathrm{EH}$ (with or without atypia), and normal endometrial findings $(p=0.04)$. On the other hand, there was no significant difference between the EC subgroups by tumor grade and non-cancerous endometrial pathology groups in terms of PLR ( $p=0.84$ ) (Tab. 3). In addition, no significant difference was detected between EC subgroups by FIGO staging and non-cancerous endometrial pathology groups in terms of NLR and PLR ( $p=0.09 ; p=0.91$, respectively) (Tab. 4).

Table 5 shows the univariate and multivariate analysis of the factors associated with endometrial cancer. In the multivariate analysis, the ORs of the NLR, and PLR were $0.9(95 \%$ $\mathrm{Cl} 0.8-1.0 ; \mathrm{p}=0.58)$, and $1.0(95 \% \mathrm{Cl} 0.9-1.0 ; \mathrm{p}=0.23)$, respec-

Table 2. Neutrophil to lymphocyte and platelet to lymphocyte ratios of the patients

\begin{tabular}{|l|l|l|l|l|l|}
\hline & $\begin{array}{l}\text { Endometrial } \\
\text { cancer }(\mathbf{n = 1 2 1})\end{array}$ & $\begin{array}{l}\text { Hyperplasia with } \\
\text { atypia }(\mathbf{n = 7 1 )}\end{array}$ & $\begin{array}{l}\text { Hyperplasia } \\
\text { without atypia } \\
(\mathbf{n = 1 2 3})\end{array}$ & $\begin{array}{l}\text { Normal endometrium } \\
\mathbf{( n = 1 1 6 )}\end{array}$ & p-value \\
\hline Neutrophil-lymphocyte ratio, median (range) & $2.4(0.7-12.2)$ & $2.1(0.01-18)$ & $2.1(0.1-148)$ & $2(0.4-6)$ & $0.04^{*}$ \\
\hline Platelet-lymphocyte ratio, median (range) & $137.6(54.2-476.6)$ & $126.8(37.5-391.8)$ & $125.2(55.1-626)$ & $133.8(15.5-363.9)$ & 0.84 \\
\hline
\end{tabular}

Table 3. Neutrophil to lymphocyte and platelet to lymphocyte ratios of the patients (EC patients divided into three groups by tumor grade)

\begin{tabular}{|c|c|c|c|c|c|c|c|}
\hline & $\begin{array}{l}\text { Grade 1-EC } \\
(n=36)\end{array}$ & $\begin{array}{l}\text { Grade 2-EC } \\
(n=63)\end{array}$ & $\begin{array}{l}\text { Grade 3-EC } \\
(n=22)\end{array}$ & $\begin{array}{l}\text { Hyperplasia } \\
\text { with atypia } \\
(n=71)\end{array}$ & $\begin{array}{l}\text { Hyperplasia } \\
\text { without atypia } \\
(n=123)\end{array}$ & $\begin{array}{l}\text { Normal } \\
\text { endometrium } \\
(n=116)\end{array}$ & p-value \\
\hline $\begin{array}{l}\text { Neutrophil-lymphocyte ratio, } \\
\text { median (range) }\end{array}$ & $2.3(1.2-7.3)$ & $2.2(0.7-12.2)$ & $2.6(0.7-6.2)$ & $2.1(0.01-18)$ & $2.1(0.1-148)$ & $2(0.4-6)$ & $0.04^{*}$ \\
\hline $\begin{array}{l}\text { Platelet-lymphocyte ratio, } \\
\text { median (range) }\end{array}$ & $\begin{array}{l}131.4 \\
(63.3-325.0)\end{array}$ & $\begin{array}{l}132.6 \\
(54.2-476.6)\end{array}$ & $\begin{array}{l}157.7 \\
(69.6-318.0)\end{array}$ & $\begin{array}{l}126.8 \\
(37.5-391.8)\end{array}$ & $\begin{array}{l}125.2 \\
(55.1-626)\end{array}$ & $\begin{array}{l}133.8 \\
(15.5-363.9)\end{array}$ & 0.84 \\
\hline
\end{tabular}

EC - endometrial cancer

Table 4. Neutrophil to lymphocyte and platelet to lymphocyte ratios of the patients (EC patients divided into early and advanced stages by FIGO staging)

\begin{tabular}{|l|l|l|l|l|l|l|}
\hline & $\begin{array}{l}\text { Stage I-II } \\
(\mathbf{n = 1 0 3 )}\end{array}$ & $\begin{array}{l}\text { Stage III-IV } \\
(\mathbf{n = 1 8})\end{array}$ & $\begin{array}{l}\text { Hyperplasia with } \\
\text { atypia }(\mathbf{n = 7 1 )}\end{array}$ & $\begin{array}{l}\text { Hyperplasia without } \\
\text { atypia }(\mathbf{n = 1 2 3})\end{array}$ & $\begin{array}{l}\text { Normal endometrium } \\
(\mathbf{n}=\mathbf{1 1 6})\end{array}$ & p-value \\
\hline $\begin{array}{l}\text { Neutrophil-lymphocyte } \\
\text { ratio, median (range) }\end{array}$ & $2.3(0.7-12.2)$ & $2.7(0.8-5.1)$ & $2.1(0.01-18)$ & $2.1(0.1-148)$ & $2(0.4-6)$ & 0.09 \\
\hline $\begin{array}{l}\text { Platelet-lymphocyte } \\
\text { ratio, median (range) }\end{array}$ & $\begin{array}{l}137.8 \\
(54.2-476.6)\end{array}$ & $\begin{array}{l}128.3 \\
(55.4-264.6)\end{array}$ & $\begin{array}{l}126.8 \\
(37.5-391.8)\end{array}$ & $\begin{array}{l}125.2 \\
(55.1-626)\end{array}$ & $\begin{array}{l}133.8 \\
(15.5-363.9)\end{array}$ & 0.91 \\
\hline
\end{tabular}

EC — endometrial cancer; FIGO — The International Federation of Gynecology and Obstetrics 
Table 5. Univariate and multivariate analysis of factors associated with endometrial cancer

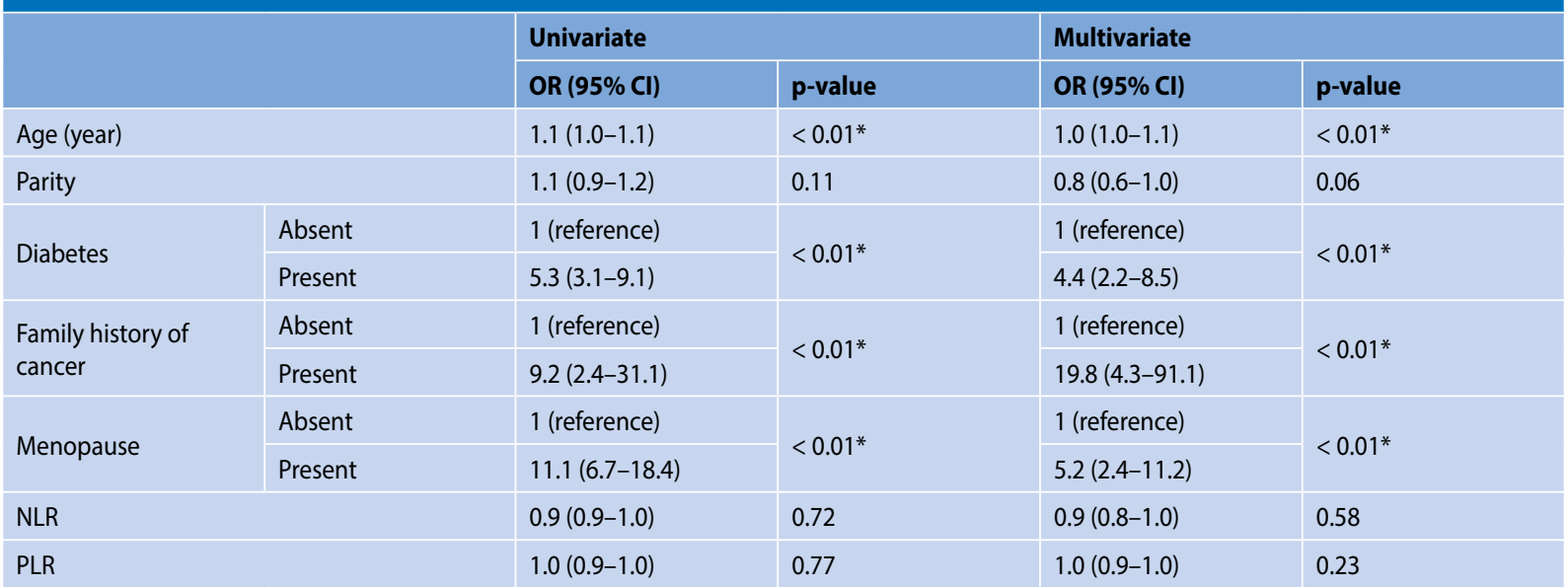

95\% $\mathrm{Cl}-95 \%$ confidence interval; NLR - neutrophil to lympohocyte ratio; OR — odds ratio; PLR — platelet to lymphocyte ratio

Table 6. Neutrophil to lymphocyte and platelet to lymphocyte ratios of the endometrial cancer patients by grouping according to tumor grade and FIGO stage

\begin{tabular}{|c|c|c|c|c|c|}
\hline & & $\begin{array}{l}\text { Neutrophil- lymphocyte ratio, median } \\
\text { (range) }\end{array}$ & p-value & $\begin{array}{l}\text { Platelet-lymphocyte ratio, median } \\
\text { (range) }\end{array}$ & p-value \\
\hline \multirow{2}{*}{ FIGO stage } & Stage I-II $(n=103)$ & $2.3(0.7-12.2)$ & \multirow{2}{*}{0.79} & $137.8(54.2-476.6)$ & \multirow{2}{*}{0.70} \\
\hline & Stage III-IV $(n=18)$ & $2.7(0.8-5.1)$ & & $128.3(55.4-264.6)$ & \\
\hline \multirow{3}{*}{ Tumor grade } & Grade $1(n=36)$ & $2.3(1.2-7.3)$ & \multirow{3}{*}{0.22} & $131.4(63.3-325.0)$ & \multirow{3}{*}{0.61} \\
\hline & Grade $2(n=63)$ & $2.2(0.7-12.2)$ & & $132.6(54.2-476.6)$ & \\
\hline & Grade $3(n=22)$ & $2.6(0.7-6.2)$ & & $157.7(69.6-318.0)$ & \\
\hline
\end{tabular}

tively. Other clinical factors including age, diabetes, family history of cancer, and menopause were found associated with endometrial cancer. Moreover, no significant association was found between NLR, PLR, and tumor grade, FIGO stages ( $p=0.22 ; p=0.61 ; p=0.79 ; p=0.70$, respectively) (Tab. 6).

\section{DISCUSSION}

In a study that investigated the blood parameters in cancer, it was found that relative lymphocytopenia secondary to the increased neutrophil count can be seen as a response to systemic inflammation. Tumor-associated neutrophils (TANS) affect the extracellular environment through enzymatic interactions that result in endothelial cell migration, increased fibroblast growth factor secretion, and migration of tumor cells. As a result of these, tumor progression and neo-vascularization occur [7, 8]. Certain studies regarding lung and anal cancer also support this finding. The result of these studies is that neutrophilia is a strong factor that predicts the poor prognosis in anal cancer and advanced stage III lung cancer $[9,10]$. In another study conducted by Tavares-Murta et al. [11], showed that neutrophilia is a good prognostic factor to predict the metastasis and recurrence in advanced stage cervical carcinoma. Besides, patients who had locally advanced cervical cancer with higher baseline lymphocyte count had better treatment responses to chemoradiotherapy [12]. NLR is accepted as an effective and simple parameter of inflammation [13]. Starting from this point of view, NLR and prognosis association has been investigated in different types of cancer [14-16]. Higher NLR association with prognosis can be explained by these: i) immune-response to the tumor is mainly via lymphocytes; ii) neutrophils are secreting the vast majority of vascular endothelial growth factor (VEGF) to the circulation which enhances the tumor progression [17]. An important study that investigates the NLR differences between endometrial pathologies revealed that NLR was higher in EC patients rather than patients with non-cancerous endometrial pathologies [18]. In another recent study that evaluates the NLR as a discriminative factor for endometrial pathologies showed that EC patients have significantly higher NLR than patients with other endometrial pathologies including endometrial hyperplasia and normal endometrial findings, however, they did not separate the endometrial hyperplasia patients as with atypia and without atypia in their study [13]. In the present study, it was determined that higher NLR is associ- 
ated with EC as compatible as the abovementioned studies, however, no difference was found between endometrial hyperplasia with atypia and endometrial hyperplasia without atypia patients in terms of NLR. In a study conducted by Cong et al. [4], ROC curve was generated to calculate a cut-off value for overall-survival (OS) in endometrial cancer patients and it was found that higher NLR was associated with poor OS rates. In the present study, ROC curve analysis was performed to detect a cut-off value to distinguish EC among groups and higher values of NLR were determined associated with cancer. Therefore, it can be said that NLR can be used to discriminate EC from other endometrial pathologies according to the findings of the present study.

Increased platelet count is another inflammation marker that occurred as a response to tumor [19]. Cytokines as a response to inflammation such as IL-1, IL-6 initiate thrombocytosis via megakaryocyte proliferation [20, 21]. Inflammation is associated with increased platelet and decreased lymphocyte levels. Decreased lymphocyte levels were showed colon cancer and pancreatic adenocancer previously. It was also shown that poor prognosis in pancreatic adenocancer is associated with decreased number of tumor-infiltrating lymphocytes [22, 23]. Thus, platelet to lymphocyte ratio becomes another marker for inflammatory processes by the combination of haematologic parameters [24]. Higher PLR was found associated with poor prognosis in non-small cell lung cancer [25]. Besides, PLR was found associated with lymphovascular space invasion (LVSI), lymph node involvement, and distant metastasis in endometrial cancer [26]. In a study conducted by Cakmak et al. [27], PLR was determined as a non-specific inflammatory marker that gives access to predict atypical EH in abnormal uterine bleeding patients, however, they did not enroll the EC patients in their study. Moreover, another crucial study that investigates the importance of platelets to discriminate endometrial pathologies showed that PLR was not significantly different between EC patients and patients with benign endometrial pathologies, however, patients with EH were not enrolled in this study [28]. In another study, PLR was found significantly higher in EC patients in comparison with patients with endometrial hyperplasia or normal endometrial findings, and this study also did not divide the patients of hyperplasia group to with and without atypia groups [29]. In the present study, PLR was determined higher in EC patients among all groups of patients. However, this difference was not found statistically significant. In the present study, there was also a small increase in PLR, which was not statistically significant, in the endometrial hyperplasia group with atypia compared to the endometrial hyperplasia group without atypia. According to the findings of the present study, PLR does not seem to be a good non-specific inflammatory marker in contrast to the NLR in terms of predicting EC or hyperplasia with atypia among endometrial pathologies.

In the previous studies which investigated the prognostic value of NLR and PLR in endometrial cancer patients, it was found that higher NLR and PLR are associated with higher FIGO stages and tumor grades [4, 30, 31]. On the other hand, no significant difference was found between FIGO stages in terms of NLR and PLR in the study conducted by Kurtoglu et al. [28]. In the present study, no significant association was found between NLR, PLR and tumor FIGO stages, tumor grades.

The retrospective design of the present study is the main limitation. However, the strenght of this study is the data homogeneity that comes from single-center experience and being conducted by the same team.

\section{CONCLUSIONS}

NLR and PLR are systemic immune response parameters that can be easily evaluated from routine blood tests with no additional cost. NLR was found significantly higher in EC among endometrial pathologies. Thus, NLR potentially might be used in the future to discriminate EC from other endometrial pathologies including endometrial hyperplasia and normal endometrial findings. PLR is not a good predictor to make discrimination among endometrial pathology groups according to present study findings. In addition to other inflammation markers to the NLR and PLR may give access to discriminate one of the pathologies among endometrial cancer, endometrial hyperplasia with atypia, endometrial hyperplasia without atypia, and pathologically normal patients. Further studies are needed to investigate the value of NLR and PLR in endometrial pathologies.

\section{Contributions}

Ol: manuscript writing, data management, data analysis. RIM: data collection and analysis. OA: project development and administration. AM: data collection and analysis. SK: supervision, review of the manuscript.

\section{Ethical approval}

This study was carried out in consensus with our university's ethics guidelines. The ethics committee approval was obtained for this study.

\section{IRB approval}

This study was carried out in consensus with our university's ethics guidelines.

\section{Conflict of interest}

The authors declare that they have no conflict of interest. 


\section{REFERENCES}

1. Babu SN, Chetal G, Kumar S. Macrophage migration inhibitory factor: a potential marker for cancer diagnosis and therapy. Asian Pac J Cancer Prev. 2012; 13(5): 1737-1744, doi: 10.7314/apjcp.2012.13.5.1737, indexed in Pubmed: 22901113.

2. Guo YZ, Pan L, Du CJ, et al. Association between C-reactive protein and risk of cancer: a meta-analysis of prospective cohort studies. Asian Pac J Cancer Prev. 2013; 14(1): 243-248, doi: 10.7314/apjcp.2013.14.1.243, indexed in Pubmed: 23534731.

3. Heller DS, Mosquera C, Goldsmith LT, et al. Body mass index of patients with endometrial hyperplasia: comparison to patients with proliferative endometrium and abnormal bleeding. J Reprod Med. 2011; 56(3-4): 110-112, indexed in Pubmed: 21542526.

4. Cong R, Kong F, Ma J, et al. Combination of preoperative neutrophil-lymphocyte ratio, platelet-lymphocyte ratio and monocyte-lymphocyte ratio: a superior prognostic factor of endometrial cancer. BMC Cancer. 2020; 20(1): 464, doi: 10.1186/s12885-020-06953-8, indexed in Pubmed: 32448185.

5. Kao SCH, Pavlakis N, Harvie R, et al. High blood neutrophil-to-lymphocyte ratio is an indicator of poor prognosis in malignant mesothelioma patients undergoing systemic therapy. Clin Cancer Res. 2010; 16(23): 5805-5813, doi: 10.1158/1078-0432.CCR-10-2245, indexed in Pubmed: 20956618.

6. Kwon $\mathrm{HC}$, Kim SH, Oh SY, et al. Clinical significance of preoperative neutrophil-lymphocyte versus platelet-lymphocyte ratio in patients with operable colorectal cancer. Biomarkers. 2012; 17(3): 216-222, doi: 10.3109/1354750X.2012.656705, indexed in Pubmed: 22424597.

7. Fridlender ZG, Sun J, Kim S, et al. Polarization of tumor-associated neutrophil phenotype by TGF-beta:'N1'versus'N2'TAN. Cancer Cell. 2009; 16(3): 183-194, doi: 10.1016/j.ccr.2009.06.017, indexed in Pubmed: 19732719.

8. Shaul ME, Fridlender ZG. Cancer-related circulating and tumor-associated neutrophils - subtypes, sources and function. FEBS J. 2018; 285(23): 4316-4342, doi: 10.1111/febs.14524, indexed in Pubmed: 29851227.

9. Schernberg A. Neutrophilia as prognostic biomarker in locally advanced stage III lung cancer. PLoS One. 2018; 13(10): e0204490, doi: 10.1371/journal.pone.0204490, indexed in Pubmed: 30304046.

10. Schernberg A, Escande A, Campo ER, et al. Leukocytosis and neutrophilia predicts outcome in anal cancer. Radiother Oncol. 2017; 122(1): 137-145, doi: 10.1016/j.radonc.2016.12.009, indexed in Pubmed: 28024835.

11. Tavares-Murta B, Mendonça M, Duarte N, et al. Systemic leukocyte alterations are associated with invasive uterine cervical cancer. Int J Gynecol Cancer. 2010; 20(7): 1154-1159, doi: 10.1111/igc.0b013e3181ef8deb, indexed in Pubmed: 21495217.

12. Choi $\mathrm{CH}$, Kang $\mathrm{H}, \mathrm{Kim} W Y$, et al. Prognostic value of baseline lymphocyte count in cervical carcinoma treated with concurrent chemoradiation. Int J Radiat Oncol Biol Phys. 2008; 71(1): 199-204, doi: 10.1016/j. ijrobp.2007.09.024, indexed in Pubmed: 18037578.

13. A scoring model based on neutrophil to lymphocyte ratio predicts recurrence of HBV-associated hepatocellular carcinoma after liver transplantation. PLoS One. 2011; 6(9): e25295, doi: 10.1371/journal. pone.0025295, indexed in Pubmed: 21966488.

14. Hasegawa $T$, Iga $T$, Takeda $D$, et al. Neutrophil-lymphocyte ratio associated with poor prognosis in oral cancer: a retrospective study. BMC Cancer. 2020; 20(1): 568, doi: 10.1186/s12885-020-07063-1, indexed in Pubmed: 32552873.

15. Hirahara T, Arigami T, Yanagita S, et al. Combined neutrophil-lymphocyte ratio and platelet-lymphocyte ratio predicts chemotherapy response and prognosis in patients with advanced gastric cancer. BMC Cancer. 2019; 19(1): 672, doi: 10.1186/s12885-019-5903-y, indexed in Pubmed: 31286873.

16. Ethier JL, Desautels D, Templeton A, et al. Prognostic role of neutrophil-to-lymphocyte ratio in breast cancer: a systematic review and meta-analysis. Breast Cancer Res. 2017; 19(1): 2, doi: 10.1186/s13058016-0794-1, indexed in Pubmed: 28057046.
17. Kusumanto Y, Dam W, Hospers G, et al. Platelets and granulocytes, in particular the neutrophils, form important compartments for circulating vascular endothelial growth factor. Angiogenesis. 2003; 6(4): 283-287, doi: 10.1023/b:agen.0000029415.62384.ba, indexed in Pubmed: 15166496.

18. Acmaz G, Aksoy H, Unal D, et al. Are neutrophil/lymphocyte and platelet/lymphocyte ratios associated with endometrial precancerous and cancerous lesions in patients with abnormal uterine bleeding? Asian Pac J Cancer Prev. 2014; 15(4): 1689-1692, doi: 10.7314/apjcp.2014.15.4.1689, indexed in Pubmed: 24641391.

19. Voutsadakis IA. Thrombocytosis as a prognostic marker in gastrointestinal cancers. World J Gastrointest Oncol. 2014; 6(2): 34-40, doi: 10.4251/wjgo.v6.i2.34, indexed in Pubmed: 24567794.

20. Araneda M, Krishnan V, Hall K, et al. Reactive and clonal thrombocytosis. South Med J. 2001; 94(4): 417-420, doi: 10.1097/00007611200104000-00012, indexed in Pubmed: 11332909.

21. Kaser A, Brandacher G, Steurer W, et al. Interleukin- 6 stimulates thrombopoiesis through thrombopoietin: role in inflammatory thrombocytosis. Blood. 2001; 98(9): 2720-2725, doi: 10.1182/blood.v98.9.2720, indexed in Pubmed: 11675343.

22. Kishi Y, Kopetz S, Chun YS, et al. Blood neutrophil-to-lymphocyte ratio predicts survival in patients with colorectal liver metastases treated with systemic chemotherapy. Ann Surg Oncol. 2009; 16(3): 614-622, doi: 10.1245/s10434-008-0267-6, indexed in Pubmed: 19130139.

23. Fukunaga $A$, Miyamoto $M, C h o Y$, et al. CD8+ tumor-infiltrating lymphocytes together with CD4+ tumor-infiltrating lymphocytes and dendritic cells improve the prognosis of patients with pancreatic adenocarcinoma. Pancreas. 2004; 28(1): e26-e31, doi: 10.1097/00006676-20040100000023, indexed in Pubmed: 14707745.

24. Smith RA, Bosonnet $L$, Ghaneh $P$, et al. The platelet-lymphocyte ratio improves the predictive value of serum CA19-9 levels in determining patient selection for staging laparoscopy in suspected periampullary cancer. Surgery. 2008; 143(5):658-666, doi: 10.1016/j.surg.2007.12.014, indexed in Pubmed: 18436014.

25. Unal D, Eroglu C, Kurtul N, et al. Are neutrophil/lymphocyte and platelet/lymphocyte rates in patients with non-small cell lung cancer associated with treatment response and prognosis? Asian Pac J Cancer Prev. 2013; 14(9): 5237-5242, doi: 10.7314/арjcp.2013.14.9.5237, indexed in Pubmed: 24175807.

26. Pergialiotis V, Oikonomou M, Damaskou V, et al. Platelet to lymphocyte and neutrophil to lymphocyte ratio as predictive indices of endometrial carcinoma: Findings from a retrospective series of patients and meta-analysis. J Gynecol Obstet Hum Reprod. 2018;47(10):511-516, doi: 10.1016/j.jogoh.2018.08.016, indexed in Pubmed: 30153505.

27. Cakmak B, Gulucu S, Aliyev N, et al. Neutrophil-lymphocyte and platelet-lymphocyte ratios in endometrial hyperplasia. Obstet Gynecol Sci. 2015; 58(2): 157-161, doi: 10.5468/ogs.2015.58.2.157, indexed in Pubmed: 25798430.

28. Kurtoglu E, Kokcu A, Celik H, et al. Platelet indices may be useful in discrimination of benign and malign endometrial lesions, and early and advanced stage endometrial cancer. Asian Pac J Cancer Prev. 2015; 16(13): 5397-5400, doi: 10.7314/apjcp.2015.16.13.5397, indexed in Pubmed: 26225684.

29. Ural ÜM, Şehitoğlu İ, Tekin YB, et al. Neutrophil-to-lymphocyte and platelet-to-lymphocyte ratios in patients with endometrial hyperplasia and endometrial cancer. J Obstet Gynaecol Res. 2015; 41(3): 445-448, doi: 10.1111/jog.12536, indexed in Pubmed: 25363740.

30. Dong $Y$, Cheng $Y$, Wang J. The ratio of neutrophil to lymphocyte is a predictor in endometrial cancer. Open Life Sci. 2019; 14: 110-118, doi: 10.1515/biol-2019-0012, indexed in Pubmed: 33817142.

31. Aoyama T, Takano M, Miyamoto M, et al. Pretreatment neutrophil-to-lymphocyte ratio was a predictor of lymph node metastasis in endometrial cancer patients. Oncology. 2019; 96(5): 259-267, doi: 10.1159/000497184, indexed in Pubmed: 30893700. 